\title{
Early assessment of patient perception of outcome in total knee replacement
}

\author{
FILIPPO BONIFORTI, ANTONIO MACAIONE, STEFANO GAGLIARDI, FRANCESCO GIANGRASSO, \\ DAVIDE DI MARZO, FRANCESCO GIACCO
}

Department of Orthopaedics, San Raffaele G. Giglio Foundation, Cefalù, Italy

\begin{abstract}
Purpose: the aim of the present study was to measure patient perception in the early period after knee replacement surgery and to correlate health status with the surgery-related outcome.

Methods: thirty-eight consecutive patients who underwent total knee replacement were evaluated before surgery; at the time Radiographic evaluation was used to assess limb alignment and the Oxford Knee Score (OKS) to assess objective outcome. Health outcome was measured with the three-level version of the EuroQol - five dimension (EQ-5D-3L) selfadministered questionnaire, a standardized instrument used to measure patient's perception of health status. Evaluation was performed before surgery, at discharge from hospital, and six weeks after surgery.

Results: the mean overall EQ-5D-3L scores were 10.23 before surgery, 8.34 at discharge from the hospital, and 6.52 at six weeks. The overall EQ-5D-3L score before surgery was greater than 8 points in 31 of the 38 patients, ranging from 5 to 8 points in 21 of the 38 patients at the discharge and greater than 8 points in six of the 38 patients six weeks after surgery. Patients with scores of over 8 points at six weeks also scored more than 10 points on the discharge assessment.

Conclusions: analysis of early patient reports of performance after TKR showed marked changes in the level of satisfaction during the first six weeks after sur-
\end{abstract}

\footnotetext{
Corresponding Author:

Filippo Boniforti, MD

Department of Orthopaedics, San Raffaele G. Giglio

Foundation

Contrada Pietrapollastra- Pisciotto, 90015 Cefalù (PA), Italy

E-mail: filippo.boniforti@gmail.com
}

gery. An EQ-5D-3L score of 8 or more at follow-up should be considered a sign that the patient has not achieved satisfaction and, accordingly, should be studied in the context of a personalized follow-up schedule in order to identify as soon as possible the causes of impairment.

Level of Evidence: Level IV, prognostic case series.

Key Words: arthroplasty, knee, perception, questionnaire, replacement.

\section{Introduction}

During the past decade, patient perception of outcome following surgery has become a growing focus of attention and is now an aspect that should be taken into account in the evaluation of clinical results $(1,2)$. Although implant survival is recognized as the most important outcome measure in knee replacement surgery, patient satisfaction is becoming a key issue for surgeons, health stakeholders and health industries (3). Among surgeons, it is a well-known fact that even though recovery after knee replacement surgery can take more than a year, most patients show signs of wellness from the early weeks after surgery (4). The aim of the present study was to measure patient perception in the early period after knee replacement surgery and to correlate health status with the surgeryrelated outcome.

\section{Methods}

From October to December 2013, patients scheduled for primary total knee replacement (TKR) at our institution, and who gave their informed consent to enter 
the study, were enrolled. Age, gender, diagnosis and comorbidities were recorded for all the patients. Comorbidities were recorded according to the American Society of Anesthesiologists (ASA) classification.

Radiographic evaluation was used to assess limb alignment and the Oxford Knee Score (OKS) (5) to assess objective outcome. The Euro-Qol - five dimension (EQ-5D) questionnaire (6), a standardized instrument used to measure health outcome, was also used. The three-level version of the EQ-5D (EQ-5D-3L) consists of two pages - the EQ-5D descriptive system and the EQ visual analogue scale (EQ VAS). The EQ-5D$3 \mathrm{~L}$ descriptive system comprises the following five dimensions: mobility, self-care, usual activities, pain/discomfort and anxiety/depression. Each dimension has three levels: no problems, some problems, extreme problems. The respondent is asked to tick the boxes to indicate his/her state of health. The EQ VAS records the respondent's self-rated health on a vertical, visual analogue scale where the endpoints are labelled 'best imaginable health state' and 'worst imaginable health state' (7). The patients were provided with the necessary forms, which had to be filled in the day before surgery, at the time of discharge from hospital, and at the six-week follow-up in the outpatient clinic.

\section{Results}

Thirty-eight consecutive patients underwent TKR during the study period. Two different surgeons performed the operations, using two different types of implant: cruciate retaining or posterior substitute. The patients ranged in age from 52 to 77 years; they comprised 22 females and 16 males. Primary osteoarthritis of the knee was the diagnosis in all them. Nine patients were in ASA class III and the other 29 in ASA class II. No major complications were recorded after surgery and all the patients followed the same standard rehabilitation protocol.

In all cases, postoperative X-ray evaluation of the knee showed limb alignment, with varus-valgus, flexionextension and slope within three degrees of neutral. All the patients had an OKS greater than 40 points before surgery. Six weeks after surgery, the OKS had fallen below 30 points in all but five of the patients.
The mean EQ-5D-3L scores before surgery were: 2.05 for mobility, 1.71 for self-care, 2.13 for activity, 2.42 for pain/discomfort, and 1.92 for anxiety/depression. At discharge from hospital they were 1.82 for mobility, 1.5 for self-care, 1.71 for activity, 1.76 for pain/discomfort, and 1.55 for anxiety/depression. At the sixweek evaluation the mean EQ-5D-3L scores were 1.21 for mobility, 1.16 for self-care, 1.42 for activity, 1.39 for pain/discomfort and 1.34 for anxiety/depression. The mean overall EQ-5D-3L scores were 10.23 before surgery, 8.34 at discharge from the hospital, and 6.52 at six weeks. Measurement of health status (EQ VAS) showed mean values of 6.11 before surgery, 6.97 at discharge from hospital and 8.13 at six weeks (Fig. 1). The overall EQ-5D-3L score before surgery was greater than 8 points in 31 of the 38 patients (Fig. 2). The EQ-5D-3L score at discharge ranged from 5 to 8 points in 21 of the 38 patients (Fig. 3). The EQ-5D$3 \mathrm{~L}$ score at six weeks after surgery was greater than 8 points in six of the 38 patients (Fig. 4). Patients with scores of over 8 points at six weeks also scored more than 10 points on the discharge assessment.

\section{Discussion}

Total knee replacement is one of the most common operations in orthopaedic surgery. Outcomes depend on implant survival. Most patient reported outcomes are documented in large numbers of patients studied

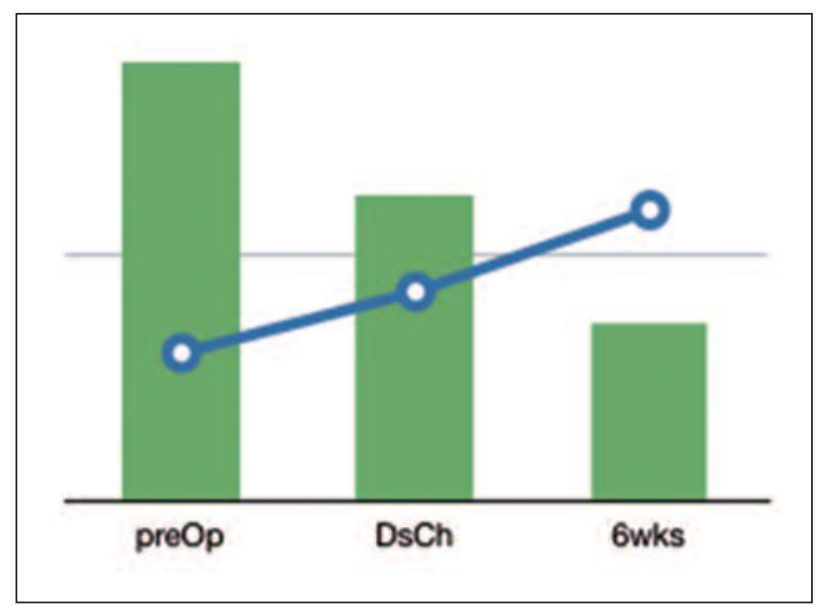

Fig. 1. EQ-5D-3L overall levels for the five dimensions (histogram) and for health status (line). 

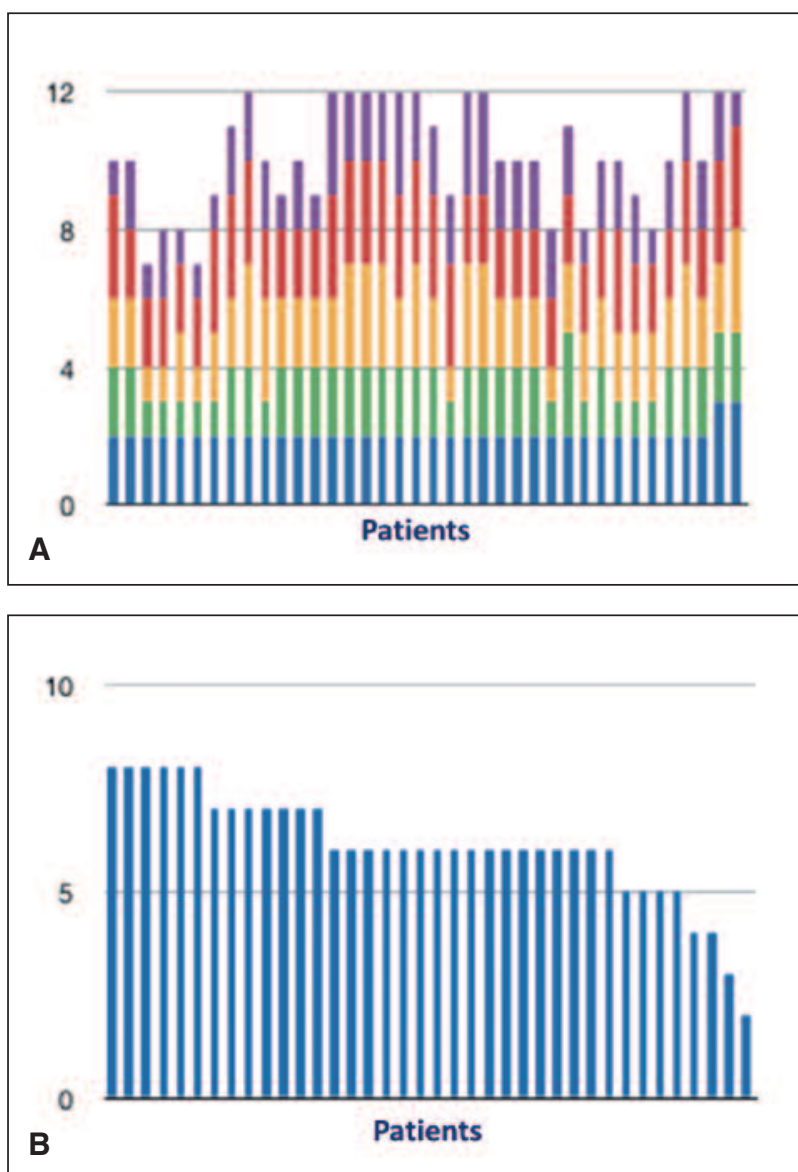

Fig. 2. The pre-operative EQ-5D-3L dimensions. A: The mean score on the descriptive system was 10.23 out of 15 points. $B$ : The mean score on the visual analogue scale for health status (EQ VAS) was 6.1 out of 10 points.

in multicenter trials. The importance of patients' perceptions has been highlighted by the National Joint Registry of England, Wales and Northern Ireland, which devoted a section of its annual report to patient reported outcome measures (PROMs) (8).

Stakeholders, health service systems, hospitals, industries and pharmaceutical brands have thus begun to use patient perception outcomes as benchmarking parameters. However, biases in the collection of PROM data in large series (lack of detail and numbers of questionnaires sent out and not returned) make assessment difficult (9).

Patient satisfaction, particularly with surgical procedures, is difficult to measure. Instruments and scales have been available for decades and the scientific community has also identified various factors contributing
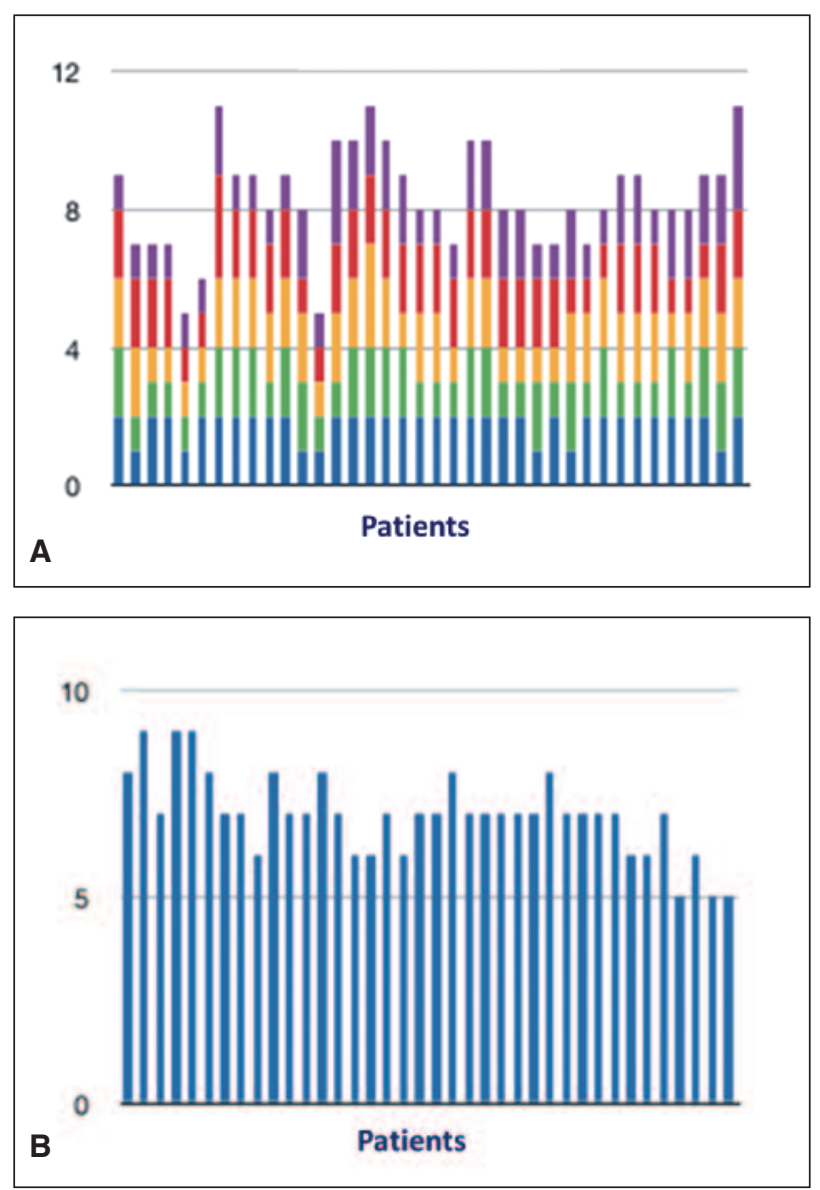

Fig. 3. The EQ-5D-3L dimensions at discharge. A: The mean score on the descriptive system was 8.34 out of 15 points. B: The mean score on the visual analogue scale for health status (EQ VAS) was 6.97 out of 10 points.

to outcome. For example, social deprivation in patients undergoing TKR is associated with significantly worse pre-operative pain and functional status and more socially deprived patients are significantly more likely to suffer a greater level of comorbidity (10). For these reasons, social deprivation is considered a strong predictor of outcome $(11,12)$.

A high level of satisfaction has been reported following joint replacement surgery (13); even though patients report a bad outcome in terms of pain and function, they may still be satisfied with the surgery $(14,15)$. In order to identify thresholds for whether or not a patient achieved a satisfactory symptom state, we considered the visual analogue scale on health status (EQ VAS) together with the surgery-related outcome level. Our study, applying a cut-off of 8 points on the EQ- 

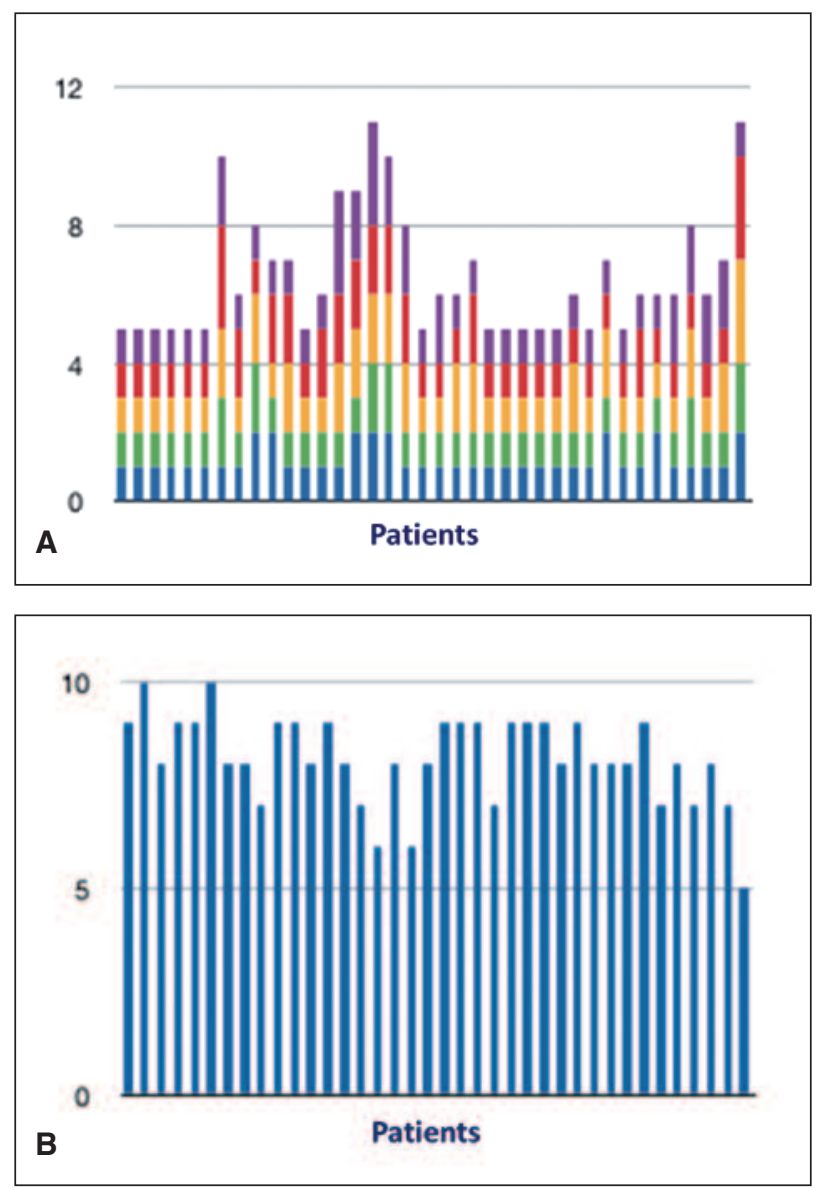

Fig. 4. The EQ-5D-3L dimensions at 6 weeks. A: The mean score on the descriptive system was 6.52 out of 15 points. B: The mean score on the visual scale for health status (EQ VAS) was 8.13 out of 10 points.

5D-3L, provides a possible means of detecting satisfaction within the six weeks following surgery; satisfaction, evaluated at single patient level, could be deemed present in patients recording a score below the measured mean value. It can be assumed that under the score of 8 points on the EQ-5D 3L the patient is in the no-problem zone. On the contrary, when the measured level is over 8 points the patient should remain under clinical supervision.

An important consideration, however, is whether six weeks is the correct length of time for assessing TKR surgery outcome from the patient's perspective. It is our opinion that six weeks, being only the beginning of the patient's recovery, is too soon to assess joint replacement outcome. Nevertheless, patients with acceptable symptoms and who are satisfied with the procedure during the early post-surgery period can probably follow a conventional rehabilitation protocol. On the contrary, patients reporting problems above the average level, i.e. in our study those scoring more than 8 points, should be put on a different program of clinical follow-up in order to identify and address the main causes of concern.

In order to define clear PROM thresholds, Keurentis et al. (16) measured Patient Acceptable Symptom State (PASS) thresholds after total hip or knee replacement. They advised against using these PASS thresholds as absolute thresholds in defining whether or not a patient has attained an acceptable symptom state after total joint replacement (16).

We suggest that although direct performance assessment and patient-reported questionnaires are both acceptable outcome measurement tools, they are complementary and should be reported as such (17). It may be helpful to use a framework of different outcome tools. From this perspective, it might be useful to consider the three levels envisaged by World Health Organization classification of impairments, disability and handicaps (18): the replacement, its function within the limb, and the functioning of the limb within the person.

In conclusion, this prospective analysis of early patient reports of performance after TKR showed marked changes in the level of satisfaction during the first six weeks after surgery. An EQ-5D-3L score of 8 or more at follow-up should be considered a sign that the patient has not achieved satisfaction and, accordingly, should be studied in the context of a personalized follow-up schedule in order to identify as soon as possible the causes of impairment.

Acknowledgments: We would like to thank Mrs Giovanna Giordano and Mrs Caterina Marte for their help with outcome database.

\section{References}

1. Dawson J, Doll H, Fitzpatrick R, Jenkinson C, Carr AJ. The routine use of patient reported outcome measures in healthcare settings. BMJ. 2010;340:c186.

2. Scott CE, Howie CR, MacDonald D, Biant LC. Predicting patient dissatisfaction following total knee replacement: a 
prospective study of 1217 cases. J Bone Joint Surg Br. 2010; 92:1253-1258.

3. Beard DJ, Knezevic K, Al-Ali S, Dawson J, Price AJ. The use of outcome measures relating to the knee. Orthopaedics and Trauma. 2010;24:309-316.

4. Judge A, Arden NK, Kiran A, et al. Interpretation of patientreported outcomes for hip and knee replacement surgery: identification of thresholds associated with satisfaction with surgery. J Bone Joint Surg Br. 2012;94:412-418.

5. Dawson J, Fitzpatrick R, Murray D, Carr A. Questionnaire on the perceptions of patients about total knee replacement. J Bone Joint Surg Br. 1998;80:63-69.

6. Devlin NJ, Parkin D, Browne J. Patient-reported outcome measures in the NHS: new methods for analysing and reporting EQ-5D data. Health Econ. 2010;19:886-905.

7. http://www.euroqol.org

8. http://www.njrcentre.org.uk/njrcentre/default.aspx

9. Clement ND, Macdonald D, Simpson AH. The minimal clinically important difference in the Oxford knee score and Short Form 12 score after total knee arthroplasty. Knee Surg Sports Traumatol Arthrosc. 2013. [Epub ahead of print]

10. Davis ET, Lingard EA, Schemitsch EH, Waddell JP. Effects of socioeconomic status on patients' outcome after total knee arthroplasty. Int J Qual Health Care. 2008;20:40-46.

11. Fortin PR, Penrod JR, Clarke AE, et al. Timing of total joint replacement affects clinical outcomes among patients with osteoarthritis of the hip or knee. Arthritis Rheum. 2002; 46:3327-3330.

12. Lingard EA, Katz JN, Wright EA, Sledge CB. Kinemax Outcomes Group. Predicting the outcome of total knee arthroplasty. J Bone Joint Surg Am. 2004;86-A:2179-2186.

13. Van der Meulen J, Lewsey J, Hardoon S, et al. National Joint Registry of England, Wales and Northern Ireland: 2nd Annual Report. 2005. http://www.njrcentre.org.uk/NjrCentre/ (date last accessed 10 October 2011).

14. Baker PN, van der Meulen JH, Lewsey J, et al. The role of pain and function in determining patient satisfaction after total knee replacement: data from the National Joint Registry for England and Wales. J Bone Joint Surg Br. 2007;89:893-900.

15. Nilsdotter AK, Petersson IF, Roos EM, Lohmander LS. Predictors of patient relevant outcome after total hip replacement for osteoarthritis: a prospective study. Ann Rheum Dis. 2003;62:923-930.

16. Keurentis JC, Van Tol FR, Fiocco M, et al. Patient acceptable symptoms states after total hip or knee replacement at midterm follow up: thresholds of the Oxford hip and knee scores. Bone Joint Res. 2014;3:7-13.

17. Hamilton DF, Gaston P, Simpson AH. Is patient reporting of physical function accurate following total knee replacement? J Bone Joint Surg Br. 2012;94:1506-1510.

18. No authors listed. World Health Organization. International Classification of impairment, disability and handicaps (ICIDH) Geneva: WHO 1980. 